\title{
Antimicrobial Stewardship at the Core of COVID-19 Response Efforts: Implications for Sustaining and Building Programs
}

\author{
Hasti Mazdeyasna ${ }^{1}$ - Priya Nori ${ }^{2}$ - Payal Patel ${ }^{3} \cdot$ Michelle Doll $^{4} \cdot$ Emily Godbout $^{4} \cdot$ Kimberly Lee $^{5} \cdot$ Andrew J. Noda $^{5}$. \\ Gonzalo Bearman $^{4} \cdot$ Michael P. Stevens $^{6}$ (D)
}

Published online: 3 July 2020

(C) Springer Science+Business Media, LLC, part of Springer Nature 2020

\begin{abstract}
We describe traditional antimicrobial stewardship program (ASP) activities with a discussion of how these activities can be refocused in the setting of the COVID-19 pandemic. Additionally, we discuss possible adverse consequences of ASP attention diversion on COVID-19 response efforts and overall implications for future pandemic planning. We also discuss ASP in collaboration with other groups within health systems and how COVID-19 may affect these relationships long term. Despite the paucity of literature on Antimicrobial Stewardship and COVID-19, the potential contributions of ASPs during a pandemic are numerous. ASPs can develop strategies to identify patients with COVID-19-like-illness; this is particularly useful when these patients are missed at the time of health system entry. ASPs can also play a critical role in the management of potential drug shortages, developing local treatment guidelines, optimizing the use of antibiotics, and in the diagnostic stewardship of COVID-19 testing, among other roles. Importantly, it is often difficult to ascertain whether critically ill patients who are hospitalized with COVID-19 have concurrent or secondary bacterial infections-ASPs are ideally situated to help optimize antimicrobial use for these patients via a variety of mechanisms. ASPs are uniquely positioned to aid in pandemic response planning and relief efforts. ASPs are already integrated into health systems and play a key role in optimizing antimicrobial prescribing. As ASPs assist in COVID-19 response, understanding the role of ASPs in pandemic relief efforts may mitigate damage from future outbreaks.
\end{abstract}

Keywords Antimicrobial stewardship · Coronavirus disease 2019 • COVID-19 · SARS-CoV-2

This article is part of the Topical Collection on Healthcare Associated Infections

Michael P. Stevens

michael.stevens@vcuhealth.org

1 Virginia Commonwealth University School of Medicine, Richmond, VA, USA

2 Department of Medicine, Division of Infectious Diseases, Montefiore Medical Center, Albert Einstein College of Medicine, 3411 Wayne Avenue 4H, Bronx, NY 10467, USA

3 Infectious Diseases Section, Ann Arbor VA Medical Center (111-i), 2215 Fuller Road, Ann Arbor, MI 48105, USA

4 Healthcare Infection Prevention Program, Virginia Commonwealth University Health System, Richmond, VA, USA

5 Virginia Commonwealth University Health System, Richmond, VA, USA

6 Healthcare Infection Prevention Program, Virginia Commonwealth University Health System, North Hospital, 1300 E. Marshall Street, P. O. Box 980019, Richmond, VA 23298, USA

\section{Introduction}

The coronavirus disease 2019 (COVID-19) pandemic has had a devastating global impact, with 6,287,771 reported cases and 379,941 deaths as of June 3, 2020 [1••]. The number of hospitalized COVID-19 patients has overwhelmed the health system infrastructure in many parts of the world [2]. Antimicrobial stewardship programs (ASPs) have not traditionally been involved in disaster relief planning or pandemic response efforts [3•]. ASPs represent an enormous potential resource, as these programs are ubiquitous, often include physicians and pharmacists with specialized infectious disease training, and often are already integrated and closely collaborated with infection prevention departments. Leveraging the knowledge and skills of ASP personnel is critical in the setting of the current COVID-19 pandemic.

Patients with COVID-19 may develop secondary nosocomial infections with bacteria and fungi as a result of critical 
illness and prolonged hospitalizations. There is high concern that antimicrobials may be overused, a fact that may be driven by the difficulty in diagnosing actual concurrent or secondary bacterial or fungal infections. Patients may receive both empiric and pathogen-directed broad-spectrum antimicrobials. Given the potential for significant antimicrobial use, ASPs play a crucial role in monitoring antibiotic use to prevent the emergence of drug resistance during the pandemic [4].

\section{Methods}

A PubMed search using the search terms "antimicrobial stewardship and COVID-19," "antimicrobial stewardship and coronavirus disease 2019," and "antimicrobial stewardship and SARS-CoV-2" was performed on April 21, 2020, resulting in only two relevant articles. In this article, we summarize the limited existing literature on this topic and discuss potential ways that ASPs can be utilized in COVID-19 response efforts. Additionally, we discuss possible adverse consequences of ASP attention diversion on COVID-19 response efforts and the overall implications for future pandemic planning.

\section{Results}

ASP core activities can be refocused on COVID-19 (Table 1).

\section{Prospective Audit with Intervention and Feedback}

ASP resources can be utilized to identify patients with COVID-19-like illness in the health system [5•]. Stevens et al. showed that they can quickly revise their existing ASP platform to identify patients with positive or pending SARS$\mathrm{CoV}-2$ polymerase chain reaction (PCR) test results [5•]. Once patients with positive SARS-CoV-2 PCR test results are identified, their medication orders and treatment plans may be audited to ensure concordance with treatment guidelines. In addition, patients with COVID-19 often receive antibiotics for suspected bacterial pneumonia [3•]. ASPs can play a critical role in the assessment of appropriateness of therapy.

A potential ramification may be diversion of focus away from non-COVID-19 patients; this may lead to subpar antimicrobial use and negative clinical consequences including the emergence of antimicrobial resistance.

In the setting of the current pandemic, ASPs have an opportunity to expand and improve upon their existing technology infrastructure. Additionally, the COVID-19 pandemic presents an opportunity for ASPs to formally integrate with infection prevention programs and enhance relationships with microbiology laboratories and information technology departments. Electronic Medical Records (EMRs) and Clinical Decision Support Systems (CDSSs) are technologies that
ASPs can enhance to optimize antimicrobial stewardship work. EMR enhancements can allow ASPs to review and intervene in the care of a higher number of patients more efficiently, while CDSSs can send real-time alerts and aid in the rapid identification of patients with positive PCR test results [8]. EMR stewardship modules can be upgraded to include audit functions for COVID19-specific medications in addition to usual antimicrobials. This may include alerts for medications requiring authorization, regimens needing dose adjustment, or flagging patients with medication contraindications.

\section{Formulary Restriction and Preauthorization}

ASPs have a critical potential role as the primary gatekeeper for the appropriate use of COVID-19 treatment medications in order to optimize patient selection and to minimize misuse. ASPs can be actively involved in the development of therapy protocols for COVID-19 and implement these through restrictions. With hydroxychloroquine being under investigation as a potential treatment of SARS-CoV-2 [9], there has been fear of medication shortage among patients with rheumatological disorders [10]. ASPs can monitor drug shortages and ensure proper allocation of medications for patients with rheumatologic conditions using formulary restrictions and preauthorization.

Early in the pandemic, other medications, many with significant potential toxicity (such as lopinavir/ritonavir), were being considered as possible COVID-19 therapies. As new data became available, the use of many of these drugs for COVID-19 has fallen out of favor. ASPs can play a key role in restricting drugs to control their optimal use and avoid toxicity.

Importantly, expanding the number of restricted drugs in the setting of the COVID-19 pandemic may cause delays in responding to antimicrobial approvals for traditional antimicrobials. In addition, relaxing some antimicrobial restriction protocols to relieve the burden of the increasing volume of COVID-19 restriction calls may lead to the suboptimal use of antimicrobials and to the emergence of antimicrobialresistant organisms.

The COVID-19 pandemic presents an opportunity for ASPs to evolve and enhance their infrastructure for addressing antimicrobial shortages.

\section{Streamlining/De-escalating Therapy and Antibiotic "Time Outs"}

Patients may be started on therapy for COVID-19, while they wait for PCR test results, especially when there are delays in testing [5•], In this setting, it is critical that therapy be reassessed and de-escalated for patients whose test results come back negative [5•]. In addition, empirical antimicrobial therapy for secondary/concomitant bacterial pneumonia 
Table 1 Selected core antimicrobial stewardship program activities and the COVID-19 pandemic

\begin{tabular}{|c|c|c|c|}
\hline $\begin{array}{l}\text { Core antimicrobial } \\
\text { stewardship activity }\end{array}$ & $\begin{array}{l}\text { How activity can be refocused on } \\
\text { COVID-19 response efforts }\end{array}$ & $\begin{array}{l}\text { Possible adverse consequences for } \\
\text { normal ASP activities }\end{array}$ & Potential long-term implications \\
\hline $\begin{array}{l}\text { Prospective audit with } \\
\text { intervention and } \\
\text { feedback }\end{array}$ & $\begin{array}{l}\text { - Create rules to identify patients with } \\
\text { COVID-19-like illness in the health } \\
\text { system [5•] } \\
\text { - Identify patients with COVID-19 and } \\
\text { review their COVID-19-focused } \\
\text { medications to make sure that these are } \\
\text { being used in guideline concordant } \\
\text { fashion and safely } \\
\text { - Review annronriateness of treatment }\end{array}$ & $\begin{array}{l}\text { - Diversion of focus away from } \\
\text { non-COVID-19 patients; this may lead } \\
\text { to suboptimal antimicrobial use with } \\
\text { subsequent worse clinical outcomes and } \\
\text { antimicrobial resistance }\end{array}$ & $\begin{array}{l}\text { - COVID-19 presents ASPs an opportunity } \\
\text { to bolster their infection technology } \\
\text { infrastructure } \\
\text { - COVID-19 presents opportunities for } \\
\text { more formal integration with infection } \\
\text { prevention programs, microbiology } \\
\text { laboratories, and information } \\
\text { technology departments }\end{array}$ \\
\hline
\end{tabular}

- Review appropriateness of treatment plans for COVID-19 patients with secondary bacterial and fungal infections [4]

- Monitor for experimental treatment toxicity (following things like the QTc for patients receiving hydroxychloroquine)

- Modify anti-infective therapy to utilize agents with less frequent dosing intervals to limit nursing exposure (e.g., ESBL E. coli treatment could be de-escalated from meropenem to ertapenem)

Formulary restriction and - ASPs can restrict medications being preauthorization considered for the treatment of COVID-19 (such as chloroquine, hydroxychloroquine, lopinavir/ritonavir, and remdesivir)

- ASPs can also monitor key antimicrobial and other drug shortages and restrict medications accordingly

Streamlining/de-escalating - ASPs can create de-escalation protocols therapy and antibiotic "time outs"

Education

IV to PO therapy for patients receiving treatment for COVID-19 and have a negative PCR test result and a plausible alternative diagnosis [5•]

- ASPs can also create de-escalation protocols for antimicrobial use targeting secondary/concomitant bacterial pneumonia

- ASPs can play a critical role in educating providers on local COVID-19 treatment protocols; this is especially true when an ASP is involved in creating and updating these guidelines

- Via their role in guidelines creation, ASPs can play a critical role in educating providers about potential medication toxicities (such as QTc prolongation with the combination of hydroxychloroquine and azithromycin)

- COVID-19 has also presented a situation where an enormous amount of data is becoming available almost daily; these data often include preprints and non-peer-reviewed articles. ASPs can play a critical role in interpreting these data for frontline providers

- ASPs can bolster long-standing IV to PO protocols in the setting of COVID-19; these could potentially shorten the duration of hospital stay and decrease hospital-acquired infections (HAIs) and IV-site infections $[6,7]$
- A focus on medications targeting COVID-19 may lead to delays in antimicrobial approvals for traditional restricted antimicrobials

- ASPs may relax long-standing restriction protocols in the setting of the pandemic, leading to the potential emergence of antimicrobial resistance

- If ASPs are actively involved in the de-escalation process, this may pull time away from traditional ASP efforts

- Interruption in daily activities and diversion of focus away from non-COVID-19 patients

- Temporary cessation of academic conferences due to pandemic challenges

- Traditional educational outreach of ASP (e.g., lectures, in-person rounds) may be interrupted

- ASP resources may be diverted from traditional IV to PO activities, particularly in non-COVID-19 patients
- ASPs can develop the local infrastructure to more nimbly address critical antimicrobial shortages

- ASPs have the potential for significant relationship building in the setting of the COVID-19 pandemic; these relationships may extend beyond the current pandemic and may help optimize local protocol uptake/utilization

- Educational platforms and dissemination processes developed during the pandemic may be utilized for disseminating information moving forward

\footnotetext{
- The COVID-19 pandemic presents opportunities for ASPs to formalize IV to PO therapy protocols
} 
Table 1 (continued)

\begin{tabular}{|c|c|c|c|}
\hline $\begin{array}{l}\text { Core antimicrobial } \\
\text { stewardship activity }\end{array}$ & $\begin{array}{l}\text { How activity can be refocused on } \\
\text { COVID-19 response efforts }\end{array}$ & $\begin{array}{l}\text { Possible adverse consequences for } \\
\text { normal ASP activities }\end{array}$ & Potential long-term implications \\
\hline & $\begin{array}{l}\text { - IV to PO protocols can often be } \\
\text { automated and can be driven by } \\
\text { pharmacy staff; this may be of great } \\
\text { value in the pandemic setting }\end{array}$ & & \\
\hline $\begin{array}{l}\text { Engagement with } \\
\text { microbiology } \\
\text { laboratories and } \\
\text { infection prevention } \\
\text { programs }\end{array}$ & $\begin{array}{l}\text { - ASPs can partner with microbiology } \\
\text { laboratories and infection prevention } \\
\text { programs for the real-time interpretation } \\
\text { of PCR test results; this can lead to } \\
\text { de-escalation of isolation precautions } \\
\text { (with conservation of personal } \\
\text { protective equipment); this also can aid } \\
\text { in the early identification of COVID-19 } \\
\text { patients to assist in optimizing } \\
\text { antimicrobial/other guideline } \\
\text { concordant therapy } \\
\text { - ASPs and microbiology laboratories can } \\
\text { create interim antibiograms to } \\
\text { understand patterns of antimicrobial } \\
\text { resistance in COVID-19 patients }\end{array}$ & $\begin{array}{l}\text { - Real-time assessment of COVID-19 PCR } \\
\text { results is labor intensive and may divert } \\
\text { attention away from usual ASP } \\
\text { activities }\end{array}$ & $\begin{array}{l}\text { - The current pandemic presents an } \\
\text { opportunity for ASPs to formally } \\
\text { engage in diagnostic stewardship } \\
\text { activities. This infrastructure will likely } \\
\text { be of use in the post-pandemic period } \\
\text { (for things like upper respiratory direct } \\
\text { pathogen panel interpretation, } \\
\text { Clostridiodes difficile test } \\
\text { interpretation) }\end{array}$ \\
\hline $\begin{array}{l}\text { Guidelines and clinical } \\
\text { pathways }\end{array}$ & $\begin{array}{l}\text { - ASPs are often involved with local } \\
\text { guideline creation and dissemination; } \\
\text { ASPs are ideally situated to help create } \\
\text { local COVID-19 treatment guidelines } \\
\text { and to disseminate these to frontline } \\
\text { providers } \\
\text { - In addition to creating } \\
\text { COVID-19-focused treatment } \\
\text { guidelines, ASPs can modify existing } \\
\text { guidelines (for things like } \\
\text { community-acquired pneumonia) to } \\
\text { optimize empiric therapy for } \\
\text { concomitant and secondary bacterial } \\
\text { infections } \\
\text { - ASPs can utilize the pandemic to } \\
\text { advocate for the adoption of creative } \\
\text { guidelines dissemination methods, such } \\
\text { as the creation of mobile/electronic apps } \\
\text { - ASPs can create or bolster infrastructure } \\
\text { to assess the extent to which guidelines } \\
\text { are being utilized and develop } \\
\text { mechanisms to feed back this } \\
\text { information to providers } \\
\text { - Clinical trials focused on COVID-19 } \\
\text { therapeutics are being adopted at many } \\
\text { institutions. ASPs can play a critical role } \\
\text { in cross-coordinating across these trials } \\
\text { and interpreting where these might } \\
\text { integrate in clinical management for } \\
\text { frontline providers }\end{array}$ & $\begin{array}{l}\text { - Creating COVID-19-focused guidelines } \\
\text { and keeping these updated and } \\
\text { disseminating these is time-consuming } \\
\text { and may divert ASP resources away } \\
\text { from usual activities } \\
\text { - Mobile app and electronic platform } \\
\text { creation is time-consuming, especially } \\
\text { at the time of initial creation } \\
\text { - Assessing guideline compliance and } \\
\text { providing feedback to providers is } \\
\text { time-consuming }\end{array}$ & $\begin{array}{l}\text { - The current COVID-19 pandemic } \\
\text { presents opportunities for increased } \\
\text { uptake of local treatment guidelines; } \\
\text { utilization of these guidelines may } \\
\text { persist beyond the pandemic } \\
\text { - Creative guidelines dissemination } \\
\text { methods (such as the adoption of mobile } \\
\text { apps) can continue to be utilized } \\
\text { post-pandemic } \\
\text { - Mechanisms to assess guidelines } \\
\text { compliance with feedback to providers } \\
\text { can be continued post-pandemic }\end{array}$ \\
\hline
\end{tabular}

should be de-escalated based on diagnostic test results and clinical reassessment.

A focus on patients with COVID-19 may pull time away from traditional ASP activities.

\section{Education}

Education is a core activity of ASPs [11]. A combination of passive education and active intervention can impact provider prescribing behavior and improve compliance with guidelines [11]. In the setting of the current pandemic, ASPs can be actively involved in educating providers on local COVID-19 treatment protocols, especially if ASPs are involved in developing these guidelines. Taking part in guideline development also equips ASPs to educate providers about potential medication toxicities (such as QTc prolongation with the combination of hydroxychloroquine and azithromycin). A unique feature of the COVID-19 pandemic is the enormous amount of new 
literature being released that includes non-peer-reviewed article preprints and sometimes news reports without formal data release. ASPs can take the lead on interpreting and communicating these data to providers treating patients on the frontlines.

Monitoring new data is labor intensive and requires a near daily commitment, and this time can divert traditional ASP attention on activities focused on patients without COVID-19.

While ASPs have relied on face-to-face educational engagement since their inception, ASPs must now adapt to new platforms to deliver educational content, such as video conferencing technology and online tools to maintain learners' connection with topics such as antibiotic spectrum and resistance mechanisms. Additionally, targeted, real-time education via other mechanisms such as email for things like updates to internal guidelines has a critical role in the setting of the current pandemic. Finally, programs that utilize electronic treatment guideline platforms (such as those using mobile "apps") can educate providers about new information via these tools, as well.

\section{IV to PO Therapy}

Conversion from intravenous (IV) to oral antimicrobial therapy in eligible patients has multiple potential advantages, including shortened duration of hospitalization, decreased hospital-acquired infections (HAIs), and decreased IV-site infections $[6,7]$. This is especially important in the setting of the COVID-19 pandemic where there is concern about a rise in HAIs [12]. Shortening the duration of hospital stay is highly valuable when many hospitals, especially those in outbreak epicenters, are facing a shortage of ICU beds.

A computerized system may be placed to identify patients who meet certain criteria and can safely undergo conversion from IV to oral therapy [6]. Nursing staff can support IV to PO conversion through optimizing documentation in the EMR, identifying patients who may qualify, and alerting pharmacy. Pharmacy staff can assess patient's eligibility and make recommendations to physicians [6]. Automatic IV to PO conversion protocols can be adopted that reduce the burden on physicians and enhance patient care. New processes that are put into place during the current pandemic may continue to be deployed after the pandemic ends.

\section{Engagement with Microbiology Laboratories and Infection Prevention Programs}

ASPs and infection prevention programs have great potential for synergy, and formal integration has multiple potential advantages $[13,14]$. ASPs can also collaborate with microbiology laboratories to act on diagnostic test results in real time. ASPs have established similar workflows with a variety of rapid diagnostic tests, including upper respiratory PCR panels [15]. ASPs can apply these workflows to SARS-CoV-2 PCR results for the timely de-escalation of isolation precautions when patients do not otherwise have a clinical syndrome consistent with COVID-19. Likewise, ASPs can assist with developing indications for SARS-CoV-2 serologic testing in hospitalized patients. Diagnostic stewardship presents an opportunity for ASPs to strengthen collaborative relationships with both microbiology laboratories and infection prevention programs. However, real-time assessment and response to COVID-19 diagnostic testing is labor intensive and might draw attention away from traditional ASP activities.

ASP relationships with Microbiology departments focused on diagnostic stewardship can continue in the post-pandemic period. Mechanisms for responding to test results in real-time can be applied to other molecular diagnostics, e.g. respiratory direct pathogen PCR panels, interpretation of Clostridiodes difficile testing, et cetera.

\section{Guidelines and Clinical Pathways}

ASPs can play a pivotal role in summarizing and updating the treatment recommendations for the evolving COVID-19 pandemic, serving as an institution's center for guidance on treatment. ASPs are frequently involved in the development of local treatment guidelines and their dissemination. This puts ASPs in an ideal position to develop local treatment protocols for COVID-19 and relay them to providers on the frontlines. Anecdotally, the three stewardship programs represented here were all directly involved in developing COVID-19 treatment protocols at our individual institutions. In addition, ASPs can modify current treatment plans to better serve patients with concomitant and secondary bacterial infections such as community-acquired pneumonia. The COVID-19 pandemic highlights opportunities to enhance guidelines dissemination to providers. ASPs can adopt creative solutions for guidelines dissemination such as the use of mobile/electronic software. Furthermore, ASPs can enhance their infrastructure to monitor and assess usage of guidelines and communicate this information back to providers on the frontlines as well as health system leadership.

ASPs can play a critical role in screening patients for COVID-19-focused clinical trials. Additionally, ASPs can assist in obtaining medications via expanded access programs through pharmaceutical companies. ASPs can also play a key role in placing clinical trial options in context via clinical guidelines development, thereby providing key information and context for frontline providers and helping to crosscoordinate direct clinical and research efforts.

Guidelines creation, upkeep and dissemination, as well as eligibility screening for experimental agents and obtaining medications via expanded access programs are timeconsuming activities and may divert attention away from traditional ASP efforts.

New mechanisms for guidelines development and dissemination can continue in the post-pandemic period. 
Additionally, heightened provider awareness and adoption of guidelines may enhance their utilization in the post-pandemic period.

\section{Conclusion}

ASPs are uniquely positioned to support hospital COVID-19 response efforts. Potential ASP activities can include identifying patients with disease, developing diagnostic and treatment protocols and disseminating these to providers, and optimizing guideline concordant treatment. The COVID-19 pandemic provides opportunities for ASPs to strengthen and enhance relationships with specialized groups within health systems, as well. However, the diversion of time away from traditional ASP efforts may have negative consequences, and these should be balanced with pandemic responsibilities as much as possible. New ASP processes and infrastructure adopted during the pandemic should continue to be utilized in the post-pandemic period.

\section{Compliance with Ethical Standards}

Conflict of Interest Hasti Mazdeyasna, Priya Nori, Payal Patel, Michelle Doll, Emily Godbout, Kimberly Lee, Andrew J. Noda, Gonzalo Bearman, and Michael P. Stevens declare that they have no conflicts of interest.

Human and Animal Rights and Informed Consent This article does not contain any studies with human or animal subjects performed by any of the authors.

\section{References}

Papers of particular interest, published recently, have been highlighted as:

- Of importance

- Of major importance

1.• World Health Organization. Coronavirus (COVID-19) [internet]. 2020 [updated 2020 June 3; cited 2020 June 3]. Available from: https://covid19. who.int/. These data from the WHO show the devastating impact of COVID-19 on the world.

2. Rodriguez-Morales AJ, Cardona-Ospina JA, Gutiérrez-Ocampo E, Villamizar-Peña R, Holguin-Rivera Y, Escalera-Antezana JP, et al. Clinical, laboratory and imaging features of COVID-19: a systematic review and meta-analysis. Travel Med Infect Dis. 2020;101623:101623. https://doi.org/10.1016/j.tmaid.2020. 101623.
3. Stevens MP, Patel PK, Nori P. Involving antimicrobial stewardship programs in COVID-19 response efforts: all hands on deck. Infect Control Hosp Epidemiol 2020;1-2. doi: https://doi.org/10.1017/ ice.2020.69. Opportunities for ASPs in COVID-19 response efforts are highlighted.

4. Clancy CJ, Nguyen MH. COVID-19, superinfections and antimicrobial development: what can we expect? Clin Infect Dis 2020; ciaa524. doi:https://doi.org/10.1093/cid/ciaa524

5. Stevens RW, Estes L, Rivera C. Practical implementation of COVID-19 patient flags into an antimicrobial stewardship program's prospective review. Infect Control Hosp Epidemiol. 2020;1-2. doi:https://doi.org/10.1017/ice.2020.133. This is an example of how an institution quickly modified its existing ASP infrastructure to respond to COVID-19.

6. Cyriac JM, James E. Switch over from intravenous to oral therapy: a concise overview. J Pharmacol Pharmacother. 2014;5(2):83-7. https://doi.org/10.4103/0976-500X.130042.

7. Béique L, Zvonar R. Addressing concerns about changing the route of antimicrobial administration from intravenous to oral in adult inpatients. Can J Hosp Pharm. 2015;68(4):318-26. https://doi.org/ 10.4212/cjhp.v68i4.1472.

8. Bremmer DN, Trienski TL, Walsh TL, Moffa MA. Role of technology in antimicrobial stewardship. Med Clin North Am. 2018;102(5):955-63. https://doi.org/10.1016/j.mcna.2018.05.007.

9. McCreary EK, Pogue JM. Coronavirus disease 2019 treatment: a review of early and emerging options. Open Forum Infect Dis. 2020;7(4):ofaa105. https://doi.org/10.1093/ofid/ofaa105.

10. Jakhar D, Kaur I. Potential of chloroquine and hydroxychloroquine to treat COVID-19 causes fears of shortages among people with systemic lupus erythematosus. Nat Med. 2020;26:632. https://doi. org/10.1038/s41591-020-0853-0.

11. Dellit TH, Owens RC, McGowan JE, Gerding DN, Weinstein RA, Burke JP, et al. Infectious Diseases Society of America and the Society for Healthcare Epidemiology of America guidelines for developing an institutional program to enhance antimicrobial stewardship. Clin Infect Dis. 2007;44(2):159-77. https://doi.org/10. 1086/510393.

12. Stevens MP, Doll M, Pryor R, Godbout E, Cooper K, Bearman G. Impact of COVID-19 on traditional healthcare-associated infection prevention efforts. Infect Control Hospital Epidemiol. 2020:1-2. https://doi.org/10.1017/ice.2020.141.

13. Dhar S, Cook E, Oden M, Kaye KS. Building a successful infection prevention program: key components, processes, and economics. Infect Dis Clin N Am. 2016;30(3):567-89. https://doi.org/10.1016/ j.idc.2016.04.009.

14. Septimus EJ. Antimicrobial resistance: an antimicrobial/diagnostic stewardship and infection prevention approach. Med Clin North Am. 2018;102(5):819-29. https://doi.org/10.1016/j.mcna.2018.04. 005.

15. Abbas S, Bernard S, Lee KB, Pakyz A, Doern C, Doll M, et al. Rapid respiratory panel testing: impact of active antimicrobial stewardship. Am J Infect Control. 2019;47(2):224-5. https://doi.org/10. 1016/j.ajic.2018.09.001.

Publisher's Note Springer Nature remains neutral with regard to jurisdictional claims in published maps and institutional affiliations. 